\title{
Interpretation of negative results in fine needle aspiration of discrete pulmonary lesions
}

\author{
A J WINNING, J McIVOR, W A SEED, O A N HUSAIN, N METAXAS \\ From the Departments of Medicine, Radiology, and Cytology, Charing Cross Hospital, London
}

ABSTRACT A retrospective analysis was carried out on a consecutive series of 181 percutaneous fine needle aspiration biopsies of discrete pulmonary lesions, in which the outcome was established in $95 \%$. In primary bronchial carcinoma the technique had a sensitivity of $86 \%$. There was no relationship between the size, location, or radiological appearance of the lesion and the incidence of false negative results. The principal reason for failure to diagnose malignancy was inadequacy of the material provided for cytological examination. For metastatic neoplasms of non-bronchial origin the procedure had a sensitivity of $42 \%$ and a significantly greater false negative rate than for primary bronchial malignancy. For the entire series $72(40 \%)$ of the procedures failed to produce a definite diagnosis, $29(40 \%)$ of these cases subsequently proving to be malignant. A positive diagnosis was established in only $16 \%$ of patients with benign conditions. Review of published reports and consideration of the role of this and other biopsy techniques in the investigation of discrete pulmonary lesions lead to the conclusion that needle aspiration biopsy seems particularly appropriate in the investigation of inoperable patients with probable bronchial carcinoma in whom sputum cytology and bronchoscopy do not yield a diagnosis.

We have reviewed published reports on percutaneous needle aspiration biopsy of discrete pulmonary lesions to establish the sensitivity of the method in the diagnosis of carcinoma. In those studies where sufficient data are provided, sensitivity (defined as true positive results expressed as a percentage of true positive and false negative results, in patients confirmed by other means to have carcinoma) varied from $77 \%$ to $90 \% .^{1-7}$ A very high proportion of the lesions were primary bronchial carcinomas.

While several of these studies have addressed themselves to the reasons for false negative results in bronchial carcinoma, few have defined the precise role of the method in the investigation of discrete lung lesions. In a consecutive series of needle aspiration biopsies with an overall sensitivity of $77 \%$ for carcinoma, we have examined the reasons for false negative results in the diagnosis of bronchial carcinoma. The sensitivity for this diagnosis in our series was $86 \%$. To determine the overall usefulness of the method, we have analysed the whole series and formulated a policy with regard to needle aspiration biopsy in the management of discrete pulmonary lesions.

Address for reprint requests: Dr A J Winning, Department of Medicine, Charing Cross Hospital, London W68RF.

Accepted 13 May 1986

\section{Methods}

PATIENTS AND DIAGNOSTIC CRITERIA

A consecutive series of 181 needle biopsy procedures, 100 of which were in patients later proved to have carcinoma of the bronchus, was examined retrospectively. We identified 14 negative results from patients with bronchial carcinoma.

The diagnosis of malignancy was based on the following criteria: (a) histological proof of malignancy at surgical biopsy or necropsy; $(b)$ progressive deterioration to death without other cause, or with growth of the primary lesion or metastatic spread of the tumour documented by a radiological or radionuclide imaging technique; $(c)$ unequivocal reduction in size of primary or secondary lesions documented by radiography or computed tomography after radiotherapy or cytotoxic chemotherapy or both.

Patients were classified as having carcinoma of the bronchus only in the absence of previous malignancy or of an alternative primary lesion at presentation or subsequently.

A diagnosis of carcinoma was excluded by a positive microbiological diagnosis or non-malignant histological diagnosis for the lesion in question, absence of malignancy at necropsy, or clear radiological resolution of the primary pulmonary lesion with clinical 
recovery maintained for at least 12 months without radiotherapy or cytotoxic chemotherapy.

\section{ASPIRATION PROCEDURE}

Aspiration was carried out with a $15 \mathrm{~cm}$ thin walled 22 gauge Chiba needle and single plane fluoroscopy. The position of the lesion was determined radiologically before aspiration, and accurate placement of the aspiration needle was confirmed during the procedure by radiological demonstration that the needle tip moved and coincided with the lesion during deep breathing. Aspiration was undertaken three or more times from different parts of the lesion. If the initial samples were excessively blood stained, extra samples were obtained. An expiration chest film was taken 15 minutes after the procedure and was checked for pneumothorax.

\section{SMEARS}

Smears were rapidly either wet fixed in alcohol for the Papanicolaou stain or air dried for staining by the May-Grünwald Giemsa technique. Needles were washed out in buffered saline solution and the cell suspensions were collected on to millipore membranes and stained by the Papanicolaou and other techniques.

The smear reports were graded as follows: grade I-benign cells only; II-reactive but benign cells (hyperplastic or inflammatory); IIR - atypical cells
Table 1 Results of needle aspiration biopsy from the consecutive series of procedures

\begin{tabular}{|c|c|}
\hline & $\begin{array}{l}\text { No }(\%) \text { of } \\
\text { procedures }\end{array}$ \\
\hline Bronchial carcinoma & $100(55 \cdot 2)$ \\
\hline $\begin{array}{l}\text { True positive } \\
\text { False negative }\end{array}$ & \\
\hline False positive $\quad 0$ & \\
\hline Primary neoplasms of non-bronchial origin & $26(14 \cdot 4)$ \\
\hline True positive & \\
\hline False negative & \\
\hline False positive $\quad 0$ & \\
\hline \multirow{5}{*}{$\begin{array}{l}\text { True negative diagnosis (eg infection) } \\
\text { Lesion not entered owing to overlying tissue } \\
\text { Procedures in patients lost to follow up } \\
\text { Specimens sent for microbiological } \\
\text { examinations alone } \\
\text { Total }\end{array}$} & $39(21 \cdot 5)$ \\
\hline & $3(1 \cdot 7)$ \\
\hline & $9(5.0)$ \\
\hline & $4(2 \cdot 2)$ \\
\hline & $181(100)$ \\
\hline
\end{tabular}

that are suspect but ambiguous; neoplasia not confidently excluded; III - evidence very strongly sug- $Z$ gestive of neoplasia, which warrants repeat biopsy if a positive diagnosis is not forthcoming by other means; IV-unequivocally malignant. The prefix $\mathrm{O}$ indicated a specimen providing insufficient cellular material or $\vec{P}$ consisting largely of blood or necrotic debris. Only grade IV was accepted as unequivocally diagnostic of ${ }^{\circ}$ malignancy for clinical purposes.

This classification was used throughout the study, and all the results are based on the original, not retro-ō spective, smear reports.

Table 2 Analysis of false negative aspirations in bronchial carcinomas

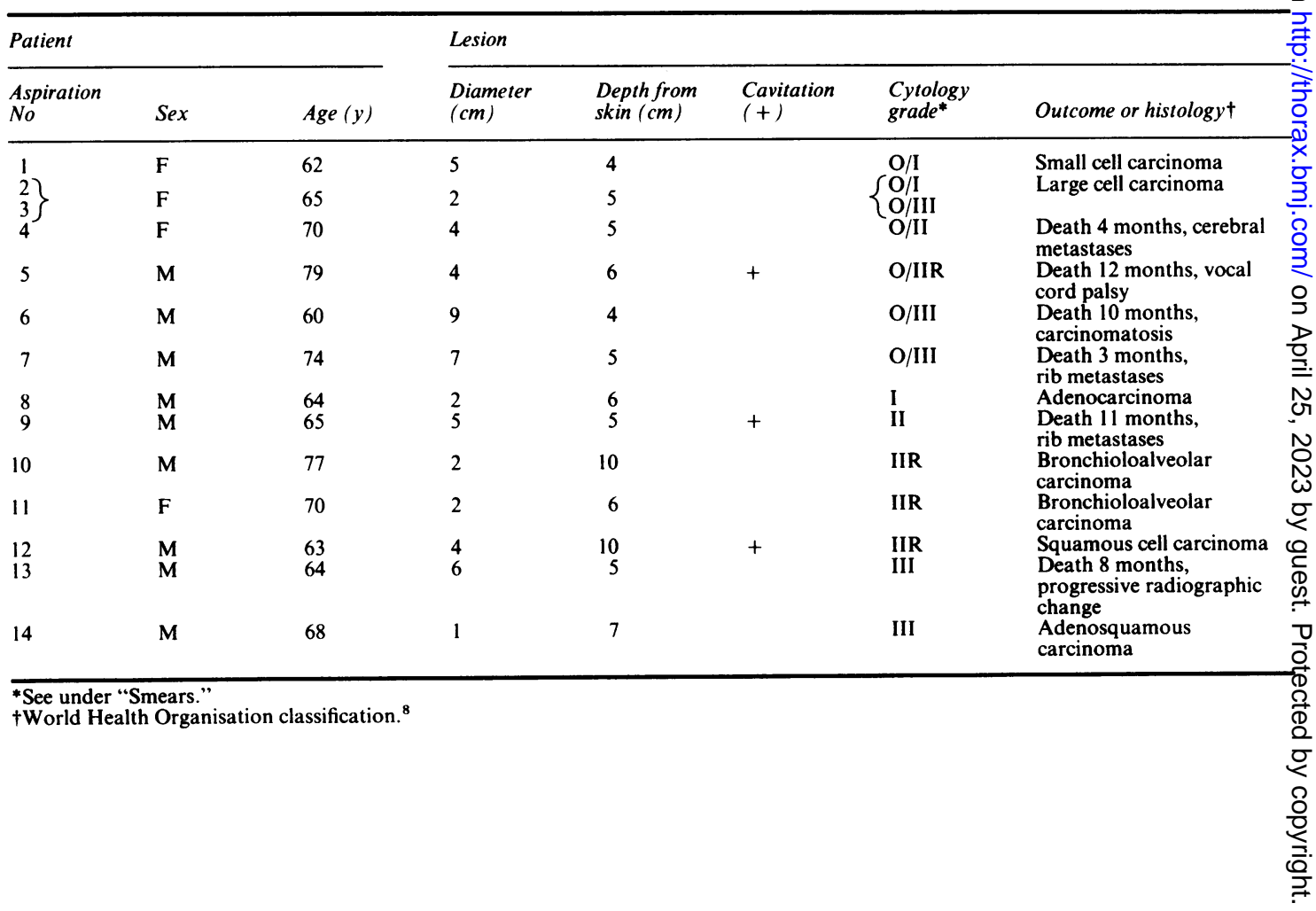


Table 3 Comparison of aspirations resulting in true positive and false negative diagnoses of bronchial carcinoma. (Values are means with standard deviations in parentheses)

\begin{tabular}{|c|c|c|}
\hline & True positive & False negative \\
\hline $\begin{array}{l}\text { Patient } \\
\text { Age (years) } \\
\text { Male:female } \\
\text { Lesion }\end{array}$ & $\begin{array}{l}68 \cdot 4(7 \cdot 8) \\
1 \cdot 7: 1\end{array}$ & $\begin{array}{l}67 \cdot 6(5 \cdot 7) \\
1 \cdot 8: 1\end{array}$ \\
\hline $\begin{array}{l}\text { Diameter }(\mathrm{cm}) \\
\text { Depth from skin }(\mathrm{cm}) \\
\text { Cavitation }\end{array}$ & $\begin{array}{l}4 \cdot 6(2 \cdot 3)^{*} \\
5 \cdot 8(2 \cdot 2)^{*} \\
23 / 86(27 \%) \dagger\end{array}$ & $\begin{array}{l}3.9(2 \cdot 3)^{*} \\
5.9(1.9)^{*} \\
3 / 14(21 \%) t\end{array}$ \\
\hline
\end{tabular}

*Difference between true positives and false negatives not significant (p $>0.05$ ) in an unpaired $t$ test.

tDifference between true positive and false negatives not significant $(p>0.05)$ in a $\chi^{2}$ test.

\section{Results}

The results of the 181 procedures are shown in table 1 . Characteristics of the patients and lesions with a false negative diagnosis of bronchial carcinoma are shown in table 2. A comparison of these with the 86 aspirations resulting in a true positive diagnosis of bronchial carcinoma is shown in table 3 . The false negative results occurred throughout the series, though only one was obtained in the initial 50 procedures. Of the true positive diagnoses, $34 \%$ were based on histological appearances, $24 \%$ on progressive deterioration to death (often with clinical features suggesting local spread), $32 \%$ on progressive deterioration to death with radiological or radionuclide evidence of growth of the primary lesion or metastatic spread of the tumour, and $10 \%$ on radiological demonstration of reduction in size of the primary or secondary lesions after radiotherapy or cytotoxic chemotherapy or both.

In four of the 39 procedures resulting in a true negative diagnosis, culture of the needle aspirate resulted in a positive identification of an infecting organism while in the remainder a non-malignant diagnosis was established by the criteria previously described (see under "Methods"). In a further 26 procedures a wide variety of neoplasms of non-bronchial origin were diagnosed histologically. These included carcinomas with nine different primary origins, Hodgkin's and non-Hodgkin's lymphomas, thymoma, teratoma, choriocarcinoma and osteosarcoma. In 11 of these cases the needle aspirate was identified as malignant. Five of the aspirates from patients subsequently lost to follow up were positive for malignancy. Three of four aspirates sent solely for microbiological examination resulted in positive cultures. In three cases the needle failed to penetrate cartilage or thickened pleura overlying the lesion. Thus $72(40 \%)$ of the total of 181 procedures failed to lead to a definite diagnosis after the investigation. These comprise the 14 false negative results in patients with bronchial carcinoma, 15 false negative results in patients with non-bronchial neoplasms, four negative results in patients lost to follow up, 35 true negative results confirmed as such by other means, three aspiration failures, and the one case in which culture of a specimen sent for microbiological examination alone proved negative.

\section{Discussion}

The results show that, with standard methods of localising lesions and aspiration, our use of fine needle aspiration achieved a sensitivity in diagnosing bronchial carcinoma very similar to that reported in other series. ${ }^{1-7}$ We can therefore expect the analysis of our false negative results to have relevance to the technique as practised in other centres.

Table 3 shows that the false negative diagnoses are not attributable to differences in the size, location within the lung, or radiological appearance of the aspirated lesions. Diagnostic yield did not significantly diminish in centrally located small lesions, as has previously been suggested, ${ }^{9}$ nor did the presence of cavitation in a lesion make a false negative result more likely.

The principal reason for failure to diagnose malignancy lies in inadequacy of the specimens. Seven of our 14 false negative results were obtained with material regarded as inadequate for cytological diagnosis. Four of the aspirates were mainly blood, two comprised minimal material, and one consisted solely of necrotic debris. In each case this occurred even though aspiration was repeated three or more times from different parts of the lesion until apparently adequate material had been obtained. We believe that this reflects an inherent limitation of the technique since it is not due to differences in the lesions.

We do not propose to comment on the sensitivity of the technique for individual types of non-bronchial neoplasm, since the small numbers and histological variety make comment on the sensitivity of the technique for individual types of tumour unreliable. The important finding for this group overall was the significantly higher false negative rate for nonbronchial than for primary bronchial malignancy $(15 / 26$ versus $14 / 100 ; p<0.001)$. Authors reporting on larger series have similarly found the sensitivity of aspiration biopsy to be lower in the diagnosis of nonbronchial malignancies, but to vary considerably between lesions of different origins. ${ }^{1310}$

Our observations on false negative results in bronchial and non-bronchial malignancy have a bearing on the problem of how to deal with negative results from this technique and the place of needle aspiration biopsy in the management of discrete pulmonary 
lesions. The 72 negative results represented $40 \%$ of the present series, and $29(40 \%)$ of these subsequently proved malignant. A similar risk of error can be found in other series. ${ }^{1-46711}$

Aspiration biopsy is usually performed for the investigation of an opacity on a chest radiograph when sputum investigations and bronchoscopy do not provide a diagnosis. We believe that it is principally applicable in patients presenting with a single discrete pulmonary opacity that is inoperable for any reason. Operable lesions, unless there are strong grounds for believing them benign, should be managed by thoracotomy and resection without prior aspiration biopsy. In those patients who prove to have malignancy such lesions will be resected anyway and a negative result has a substantial risk of error. In the patient who is inoperable, however, our experience, supported by that of others, suggests the following role for needle aspiration biopsy. If it is thought possible that the lesion is a primary bronchial carcinoma aspiration biopsy should be performed. It has a high sensitivity and a positive result has had a $100 \%$ predictive value (no false positive results) in the present and other series. ${ }^{35711}$ In view of the high sensitivity of the technique and the low morbidity ${ }^{12}$ associated with it, repeat aspiration is advisable where the initial aspirate is inadequate. It is also very desirable in all other negative cases, particularly when the first aspirate contains ambiguous or suspicious cells (grade IIR or III in our classification). These two criteria alone encompass 12 of our 14 false negative results (table 2). In theory, repeat aspiration with an $86 \%$ sensitivity would be expected to yield $98 \%$ sensitivity overall for carcinoma, leaving only $2 \%$ of patients with bronchial carcinoma undiagnosed. This specific point was not addressed in the present series, in which the procedure was repeated in only two cases. Published reports make it clear, however, that although repeat aspiration in negative cases increases the yield it has a lower sensitivity $(35-38 \%)$ than the initial aspiration. ${ }^{2613}$ Thus for patients in whom two aspiration procedures have given negative results the technique has little more to offer.

There is a further group of inoperable patients, in whom the pulmonary lesion is clinically thought unlikely to be a primary bronchial neoplasm. In the present series this included patients with pre-existing malignancy of non-bronchial origin and patients with a high probability of benign (for example, infective) lesions. Needle aspiration biopsy has less to offer in this group. In the present series the technique had a sensitivity of $42 \%(11 / 26)$ in malignant lesions of nonbronchial origin and $16 \%(7 / 43)$ in benign conditions. The results for both these types of lesion are similar to those obtained in other series. ${ }^{1310}$ Alternative techniques include bronchoscopic transbronchial lung biopsy under fluoroscopic control ${ }^{1415}$ and per cutaneous Trucut needle biopsy. ${ }^{16}$ Both techniques? have a higher yield with benign lesions than needlee aspiration biopsy. The fibreoptic technique is proba $\frac{\bar{y}}{\bar{n}}$ bly not superior to needle aspiration in non-bronchia malignancies, but very limited experience with Trucu尺 biopsy is encouraging. This latter technique, howevero is less useful for lesions below $2 \mathrm{~cm}$ in diameter, ${ }^{16-18}$. causes appreciable morbidity with more deeply placed lesions, ${ }^{161920}$ and requires considerable expertise forw its safe use.

We conclude that needle aspiration biopsy of dis $\stackrel{\vec{x}}{\vec{x}}$ crete pulmonary lesions is a highly sensitive technique for the diagnosis of primary bronchial carcinoma. It produces lower diagnostic yields in patients with metastases from non-bronchial neoplasms and with benign disease. It is particularly appropriate for use in inoperable patients in whom the pulmonary lesion is likely to be primary bronchial carcinoma.

We are grateful to Dr Bernard Fox for review of some histological material, and to Miss Katy Ferguson fo: expert typing of the manuscript.

\section{References}

1 Lalli AF, McCormack LJ, Zelch M, Reich NE Belovich D. Aspiration biopsies of chest lesions Radiology 1978;127:35-40.

2 Stevens GM, Weigen JF, Lillington GA. Needleำ aspiration biopsy of localized pulmonary lesions with amplified fluoroscopic guidance. Am J Roentgeno $\bar{\Phi}$ 1968;103:561-71.

3 Gibney RTN, Man GCW, King EG, leRiche J. Aspi ration biopsy in the diagnosis of pulmonary disease? Chest 1981;80:300-3.

4 Lees WR, Hall-Craggs MA, Manhire A. Five yearso experience of fine-needle aspiration biopsy: 454 con secutive cases. Clin Radiol 1985;36:517-20.

5 Allison DJ, Hemingway AP. Percutaneous needle biops of the lung. Br Med J 1981;282:875-8.

6 Poe RH, Tobin RE. Sensitivity and specificity of needle biopsy in lung malignancy. Am Rev Respir Dis 1980;122:725-9.

7 Veale D, Gilmartin JJ, Gibson GJ, Sumerling MDD Wadehra V. Test characteristics and prior assessment. in diagnosis of malignancy by fine needle aspiration [abstract]. Clin Sci 1985;69:84P.

8 World Health Organisation. Histological typing of lung tumours. 2nd ed. (International histologicał classification of tumours. No 1). Geneva: WHO, $1981^{\omega}$

9 Berquist TH, Bailey PB, Cortese DA, Miller WE. Trans thoracic needle biopsy: accuracy and complications iq relation to location and type of lesion. Mayo Clin Proळ 1980;55:475-81.

10 Thornbury JR, Burke DP, Naylor B. Transthoracico needle aspiration biopsy: accuracy of cytologic typing of malignant neoplasms. Am J Roentgenol $1981 \mathrm{D}$ 136:719-24. 
11 Sargent EN, Turner AF, Gordonson J, Schwinn CP, Pashky O. Percutaneous pulmonary needle biopsy. Am J Roentgenol 1974;122:758-68.

12 Sinner WN. Complications of percutaneous transthoracic needle aspiration biopsy. Acta Radiol (Diagn) 1976;17:813-27.

13 Sinner WN. Pulmonary neoplasms diagnosed with transthoracic needle biopsy. Cancer 1979;43:1533-40.

14 Stableforth DE, Knight RK, Collins JV, Heard BE, Clarke SW. Transbronchial lung biopsy through the fibreoptic bronchoscope. $\mathrm{Br} J$ Dis Chest 1978;72: 108-14.

15 Mitchell DM, Emerson CJ, Collins JV, Stableforth DE. Transbronchial lung biopsy with the fibreoptic bronchoscope: analysis of results in 433 patients. $\mathrm{Br} J \mathrm{Dis}$ Chest 1981;75:258-62.
16 Harrison BDW, Thorpe RS, Kitchener PG, McCann BG, Pilling JR. Percutaneous Trucut lung biopsy in the diagnosis of localised pulmonary lesions. Thorax 1984;39:493-9.

17 Aronovitch M, Chartier J, Kahana LA, Meakins JF, Groszman M. Needle biopsy as an aid to the precise diagnosis of intrathoracic disease. Can Med Ass J 1963;88: 120-7.

18 Janower ML, Land RE. Lung biopsy. Bronchial brushing and percutaneous puncture. Radiol Clin North Am 1971;9:73-83.

19 Adamson JS, Bates JH. Percutaneous needle biopsy of the lung. Arch Intern Med 1967;119:164-9.

20 Zavala DC, Bedell GN. Percutaneous lung biopsy with a cutting needle. Am Rev Respir Dis 1972;106:186-93. 